\title{
EL EMPRENDIMIENTO COMO CAMPO DE ESTUDIO EN LAS INSTITUCIONES DE EDUCACIÓN SUPERIOR: UNA APROXIMACIÓN DESDE LOS POSTULADOS DE MORIN Y HABERMAS ${ }^{\star}$ \\ ENTREPRENEURSHIP AS A STUDY FIELD \\ IN HIGHER EDUCATION INSTITUTIONS: AN APPROACH FROM MORIN AND HABERMAS POSTULATES
}

Recibido: 11 de septiembre de 2018

Evaluado: 2 de noviembre de 2018

Aprobado: 13 de enero de 2019

\author{
Yancira Moreno Mahecha* \\ Universidad Santo Tomás \\ Orcid: https://orcid.org/0000-0003-0962-7187
}

Cómo citar este artículo: Moreno Mahecha, Y. (2019). El emprendimiento como campo de estudio en las instituciones de educación superior: una aproximación desde los postulados de Morin y Habermas. Revista Estrategia Organizacional, 8(1). doi: https://doi. org/10.22490/25392786.3177

* $\quad$ Artículo de investigación. Consolidado desde el proyecto "La gestión organizacional y educativa: implementación del emprendimiento en el interior de las IES de Bogotá", desarrollado en el Doctorado en Educación de la Universidad Santo Tomás, Bogotá, Colombia.

** Estudiante del Doctorado en Educación de la Universidad Santo Tomás; magíster en Educación, línea Política y Sociedad, Pontificia Universidad Javeriana; especialista en Pedagogía y Docencia Universitaria, Universidad La Gran Colombia; economista de la Universidad Piloto de Colombia. Actualmente se desempeña como directora del Centro de Emprendimiento, Innovación y Desarrollo Empresarial Agustiniano (CEiDEA), de Uniagustiniana. Correo electrónico: yanciramoreno@ustadistancia.edu.co 


\section{RESUMEN}

El presente artículo aborda al emprendimiento como campo de estudio, y para ello postula nuevas perspectivas de abordaje: la complejidad y la transdisciplinariedad desde Morin, y los intereses y la acción comunicativa desde Habermas. En este horizonte, plantea por qué el emprendimiento no debe ser pensado solo desde su orientación hacia el empresarismo, sino desde una visión en conjunto que reconozca la responsabilidad social, ambiental y humanística que su ejercicio conlleva. Comprender al emprendimiento como campo de estudio busca favorecer el análisis de una problemática actual observada en la gestión de la formación en emprendimiento en las instituciones de educación superior. Se evidencia un vacío en su concepción sistémica, de ahí que se planteen varias dimensiones de análisis tanto del concepto en sí mismo como de sus implicaciones para el modelo de formación y de gestión en emprendimiento en las universidades colombianas.

Palabras clave: emprendimiento, administración, campo, teoría de la complejidad, transdisciplinariedad, gestión, acción comunicativa.

\section{ABSTRACT}

The article deals with entrepreneurship as a field of study, and for this, it postulates new perspectives of approach: complexity and transdisciplinarity from Morin, and the interests and the communicative action from Habermas. In this regard, it raises the subject of why entrepreneurship should be thought of from its orientation towards entrepreneurship and from a joint vision that recognises the social, environmental and humanistic responsibility that its exercise entails. Understanding entrepreneurship as a field of study seeks to favour the analysis of a current problem observed in the management of entrepreneurship training in higher education institutions. There is evidence of a vacuum in its systemic conception, hence several dimensions of analysis of both the concept itself and its implications for the model of entrepreneurship training and management in Colombian universities.

Keywords: Entrepreneurship, Field, Administration, Complexity Theory, Transdisciplinarity, Communicative Action. 


\section{INTRODUCCIÓN}

El origen del emprendimiento puede rastrearse desde múltiples procesos históricos y concepciones disciplinares. Desde lo primero, el emprendimiento constituye un conjunto de prácticas y experiencias que el ser humano desarrolla con el propósito de crear algo nuevo, de dar solución a una necesidad específica, de tomar nuevos rumbos antes no explorados. Por eso, podría afirmarse que, en paralelo a la historia de la humanidad, está la historia del emprendimiento, toda vez que el ser humano ha creado múltiples artefactos, técnicas, formas de organización, iniciativas, exploraciones, entre otras, que materializan un espíritu de transformación cultural y personal en procura del desarrollo individual y social (Duarte y Ruiz, 2009).

Desde lo segundo, es decir, las concepciones disciplinares, se abre un camino para el análisis del emprendimiento como objeto y campo de estudio. Aunque este horizonte es relativamente nuevo en comparación con el de otras disciplinas, y ciertamente tiende a depender de los discursos de las ciencias económicas y administrativas, no por ello deja de constituir un conjunto de presupuestos, teorías y prácticas con notorias particularidades epistémicas. Esta perspectiva, acá privilegiada, permite deslindar temporal y conceptualmente los discursos del emprendimiento, que empiezan a tomar forma incipiente en el siglo XVIII, derivados especialmente de tratados económicos y comerciales, hasta llegar a un estado actual en el que proliferan estudios y aproximaciones que permiten hablar de un campo en constitución y admite nuevos modos de abordaje y problematización (Matiz, 2009; Osorio, Gálvez y Murillo, 2010).

Los orígenes del emprendimiento como fenómeno histórico y social se encuentran en el proceso mismo de la creación de empresa. Para algunos, este es un tema joven, pero, en realidad, es rastreable en producciones teóricas del siglo XVIII y XIX, entre las que se destacan las de los economistas clásicos (Cuervo, Ribeiro y Roig, 2006). La creación de la empresa como instrumento tenía por fin armonizar los factores productivos y orientarlos hacia la práctica de elaboración de bienes y servicios.

En términos sintéticos, la génesis del término emprendedor puede encontrarse en la del término francés entreprendre, luego traducido al inglés como entrepreneur. El economista franco-irlandés Richard Cantillon (1755), en su obra Ensayo sobre la naturaleza del comercio en general, acuñó por escrito, en el siglo XVIII, el término en mención. Destaca la acción de riesgo que muchos comerciantes y empresarios europeos solían o podían realizar para hacer que un producto artesanal pudiera venderse luego a un precio mayor. El también economista francés 
Jean-Batiste Say teorizó en esta misma línea a comienzos del siglo XIX: trazó una separación importante entre el capitalista y el emprendedor. Este último fue considerado como un trabajador de especial importancia por ser capaz de enlazar diferentes medios y factores de producción para crear un nuevo producto.

En un principio, según argumenta Taylor (1961), estas ideas sobre el emprendimiento estaban relacionadas con la administración de los factores productivos $-y$, por ende, la administración de la unidad llamada empresabuscando un beneficio económico para el dueño de los medios de producción. Sin embargo, un cambio en tal paradigma empezó a gestarse desde el momento mismo en que emergieron las propuestas de Schumpeter (1963), economista austro-estadounidense que sostuvo que, en un sistema empresarial, existen dos tipos de comportamiento en las personas. Por un lado, el sujeto se identifica como emprendedor, es decir, se caracteriza por introducir nuevos productos y modos de producción, por propiciar la apertura de nuevos mercados y reorganizar la estructura empresarial. Por otro lado, la persona que efectúa acciones en condición de asalariada, está menos propensa a asumir riesgos y busca realizar una actividad dentro en la empresa a cambio de cierta remuneración económica.
A partir de los aportes de Schumpeter (1963), en la economía y en la administración de empresas se identifica un nuevo fenómeno de estudio el entrepreneurship -traducido al español como emprendimiento-, que alteró los principios planteados en relación con los factores clásicos de producción, puesto que, además del ya tradicional tema de tierra, capital y trabajo, se incluye ahora la capacidad emprendedora. En este escenario, es posible que las sociedades entiendan que, aunque no poseen determinados factores económicos de producción, sí cuentan con la capacidad emprendedora de su población para alcanzar altos niveles de desarrollo y en la calidad de vida. Girón (2000) sintetiza muy bien las contribuciones de Schumpeter en el pensamiento económico:

La visión del empresario emprendedor y del papel de la innovación en el desenvolvimiento económico ofreció nuevas posibilidades al desarrollo de la historia económica, al mismo tiempo que permitió articular una concepción cerrada al mecanicismo acumulativo o gradualista, evolutivo e incluso darwiniano en el análisis dinámico del desenvolvimiento económico. (p. 1080).

Schumpeter introduce al emprendedor en el sistema capitalista, pero no con el objeto de que se ajuste pasivamente a él, sino para que 
logre "revolucionar" las formas de producción allí insertas. La posibilidad de riqueza también está presente, pero ahora esta será el fruto del modo en que logre cristalizarse la acción emprendedora.

Ahora bien, múltiples características del emprendedor identificadas por este autor solo son susceptibles de desarrollo actual a través de un proceso organizativo dinamizado en una sociedad del conocimiento. Este será un aporte central de la obra de Drucker (1958, 1999), desde donde puede entenderse que, en las dinámicas contemporáneas, un conjunto de saberes y disciplinas influyen en el desarrollo de un emprendimiento. Es decir, la efectividad de este $-y$, desde luego, su conceptualizaciónse proyecta en una dinámica interdisciplinaria.

En sintonía con esta premisa, dimensionar el emprendimiento desde una sociedad del conocimiento solo puede hacerse posible a través de una formación que, desde temprana edad y hasta los niveles de educación superior, logre despertar las características, competencias y espíritu emprendedores. Tal propósito implica un alto grado de interconexiones disciplinares y discursivas, máxime en una sociedad como la contemporánea, donde prolifera la información, la circulación global del conocimiento es un rasgo distintivo y la fronteras físicas y virtuales tienden a desdibujarse o cointegrarse
(Morin, 2010; Rodríguez, 2006). La tradicional formación impartida en la educación media y superior, centrada en herramientas del saber técnico -contabilidad, matemática financiera y teoría administrativa-, ahora debe asegurar un giro hacia el desarrollo de las habilidades y destrezas del ser emprendedor -creatividad e innovación-, por medio de un trabajo transdisciplinar, complejo, basado en intereses específico y vinculado a las realidades y necesidades contextuales.

Conviene señalar en este punto, para el caso concreto de Colombia, que un nuevo panorama de acción surge con la Ley 1014 de 2006, más conocida como Ley de Fomento a la Cultura del Emprendimiento, que define emprendimiento de esta manera:

\begin{abstract}
Una forma de pensar, razonar y actuar centrada en las oportunidades, planteada con visión global y llevada a cabo mediante un liderazgo equilibrado y la gestión de un riesgo calculado, su resultado es la creación de valor que beneficia a la empresa, la economía y la sociedad.
\end{abstract}

Si se analiza el desarrollo y aplicación de este concepto en lo concerniente a la importancia de la creación de valor, pero también frente al desmedido afán de generar riqueza en beneficio propio, se reconoce un vacío de 
gestión organizacional y concepción sistémica en algunas instituciones de educación superior, manifestado en la forma de orientar la formación en emprendimiento. Aunque en varias instituciones de educación superior se han establecido centros de emprendimiento, en otras tan solo se considera el ejercicio de la acción emprendedora y los resultados como una función de la oficina de proyección social o del programa de administración de empresas y negocios. En otros casos no hay cátedras de formación en emprendimiento, pues se argumenta que tal proceso no debe ser curricularizado.

\section{NUEVAS PERSPECTIVAS DE ANÁLISIS DEL EMPRENDIMIENTO COMO CAMPO DE ESTUDIO EN LAS INSTITUCIONES DE EDUCACIÓN SUPERIOR}

Una tesis central estará presente a lo largo de este artículo: el emprendimiento se puede abordar y justificar como campo de estudio. El Ministerio de Educación Nacional (MEN) y el Instituto Colombiano para el Fomento de la Educación Superior (ICFES) (2011) conciben un campo de estudio como aquel que está constituido por diversas disciplinas y regiones que aportan conceptos, métodos, procedimientos, epistemologías y términos en la definición de sus discursos y sus prácticas.
Como se ha señalado, en el presente se hace necesario abordar el estudio del emprendimiento desde los temas atinentes a su conceptualización, orientación y desarrollo, pues se ha evidenciado que el mero carácter de empresarismo no basta para enfocarlo teóricamente en el proceso de formación y, más aún, para concebirlo como acción integral. Ciertamente, conviene señalar, con los cambios sociales se ha ido desvaneciendo la concepción humanista de la acción emprendedora $-y$, claro está, de la economía en general-, hasta cristalizar en una perspectiva de generación neta de riqueza.

Schumpeter (1963) sostiene que el emprendedor se distingue por dos características esenciales: ser innovador - producto y proceso- y ser creativo - mercado y organización-. Sin embargo, todavía el avance en el conocimiento no ha dado una explicación clara sobre el origen y la activación de estas características en la generalidad de las personas emprendedoras. Schumpeter (1963) ya tenía en mente este último problema:

La aplicación de cualquier mejora es una tarea completamente diferente de su invención, y que requiere aptitudes distintas. Si bien los empresarios pueden ser inventores, como pueden ser capitalistas, lo son por coinciden- 
cia y no por naturaleza, y viceversa. Además, las innovaciones que llevarán a la práctica los empresarios no precisan ser invenciones en forma alguna. (p. 98).

Schumpeter (1963) argumentará que la innovación con éxito requiere de un acto de la denominada voluntad, mas no del intelecto. Se podría decir que la innovación depende del liderazgo, y no de la inteligencia, más aún si se tiene en cuenta que no debe confundirse con el invento. Estando de acuerdo con este principio, se podría decir que la innovación es un acto creativo en la persona que le permitirá amoldarse y responder a los diferentes cambios que el entorno le va presentando.

En la actualidad, los estudios en el campo del emprendimiento difieren acerca del origen y el despertar de las características del ser emprendedor, debido a que se concentran en explicar el resultado que casi siempre se piensa como la generación de empresa. No obstante, no abordan explícitamente de dónde surgen los seres emprendedores, es decir, no explican qué factores del entorno familiar, social o educativo influyen para construir un ser emprendedor.

Desde la perspectiva de Cuervo, Ribeiro y Roig (2006), el emprendimiento es el motor del desarrollo de una sociedad. De ahí que sea lógico que la formación en emprendimiento desde las instituciones de educación superior procure trascender la idea de que el emprendimiento, muchas veces asociado a la generación de valor, sirva para propiciar el interés individual -como lo haría un empresario tradicional-, y no tanto una acción colectiva y con sentido humanizante.

Esta comprensión, sin embargo, no es nueva. En los planteamientos del filósofo y economista clásico Adam Smith se privilegia una construcción de la sociedad en perspectiva individualista, aunque ello esté en el horizonte de transformar realidades que no le satisfacen al ser humano:

El deseo de mejorar nuestra condición, [...] aunque generalmente sea sereno y desapasionado, nos acompaña desde el nacimiento hasta la tumba. En todo el intervalo que separa estos dos momentos es difícil encontrar un solo instante en que un hombre esté perfectamente y completamente satisfecho con su situación de forma que no desee cambios o mejoras de algún tipo. (Smith, 1987, p. 398).

¿Por qué el actuar del ser humano ante su propia insatisfacción toma la forma del egoísmo? En términos simples, la simpatía sería la facultad humana que frenaría al humano egoísta, en tanto la competencia sería aquello que lo potencia. En la obra de Smith podría asociarse el 
ser emprendedor al ser productivo que propicia la competencia. Por otro lado, desde la teoría económica clásica y sus representantes, como David Ricardo o Adam Smith, los factores de producción -tierra, capital y trabajo- orientaron el desarrollo de las sociedades, pero empezaron a enfrentarse retos complejos:

La capacidad de crecimiento de la población es [...] tan superior que el aumento de la especie humana solo puede mantenerse a nivel de los medios de subsistencia mediante la acción constante de la terrible ley de la necesidad, que actúa como un freno sobre la mayor capacidad de reproducción. (Malthus, 1970, p. 21).

Ekelund y Hébert (1992) explican que tal dinámica empezó a configurar múltiples tensiones y dilemas: en lo económico, lo social, lo ambiental. Si la población tiende a aumentar con mayor rapidez que la suma de los recursos para satisfacer sus necesidades, entonces hay que hacerse preguntas esenciales frente al propio sistema económico y el papel que allí ha desempeñado el afán de competencia. En este sentido, el emprendimiento, además de surgir como mecanismo de innovación, en el presente, está llamado a hacerle frente a ambas visiones: la de pensar que la obtención de riqueza puede ir en contravía de la retribución social, y la que concibe el desarrollo económico superpuesto a otros aspectos como lo humano, lo social y lo ambiental.
En los próximos párrafos se perfilará cómo el emprendimiento requiere nuevas aristas de análisis, conceptualización y desarrollo en el contexto específico de las instituciones de educación superior. La primera de ellas será la concepción del emprendimiento desde la complejidad y la transdisciplinariedad; la segunda será la de Habermas en lo concerniente a su teoría de los intereses y la acción comunicativa.

\section{UNA MIRADA AL EMPRENDIMIENTO DESDE LA TRANSDISCIPLINARIEDAD}

En la formación para el emprendimiento, en el presente, se requiere poner a circular los conceptos del "vivir en el afecto, en la amistad y en comunión con los demás y con la naturaleza" (Morin, 1996), pues el emprendimiento está también asociado a cierto conocimiento del ser interior -las potencialidades y habilidades del sujeto- y al reconocimiento del entorno, en función de generar propuestas razonables y pertinentes para el bienestar común.

La transdisciplinariedad sugiere una correlación entre distintas disciplinas y saberes; en la educación superior ha adquirido fuerza discursiva, pues el mundo real es integral, o bien, ya no basta una mirada unidisciplinar para abordar un problema. Morin (2010) señala a este respecto:

El objeto de la disciplina será entonces percibido como una cosa en sí; las relaciones y 
solidaridades de este objeto con otros, tratados por otras disciplinas, serán dejadas de lado, así como también las ligazones y solidaridades con el universo del cual el objeto es parte. La frontera disciplinaria, su lenguaje y sus conceptos propios van a aislar a la disciplina en relación con las otras y en relación con los problemas que cabalgan las disciplinas. El espíritu hiperdisciplinario va a devenir en un espíritu de propietario que prohíbe toda incursión extranjera en su parcela del saber. (p. 10).

Para poner un ejemplo, un odontólogo puede abrir su consultorio y poseer un saber técnico y especializado. Pero si requiere realmente, desde su libertad de acción y negocio, triunfar como empresario en el sector salud, deberá hacer acopio de otros saberes y herramientas técnicas: cómo contratar, cómo llevar la contabilidad, cómo hacer mercadeo, cómo actuar en lo administrativo, cómo gestionar el talento humano, entre otras. Para el objeto que atañe a este artículo, la transdisciplinariedad en el emprendimiento permite superar la mirada exclusiva desde el empresarismo. De ahí que en la actualidad sea pertinente que, en cualquier colegio, instituto técnico o institución de educación superior, se promueva cierto tipo de formación que privilegie un emprendimiento articulado a valores y características y complemente actitudes y competencias de un emprendedor concebido de manera integral.
De hecho, el perfil de las nuevas generaciones de emprendedores va a depender de las acciones de formación en el contexto de las familias y, por supuesto, de las instituciones de educación en todos los niveles. En concreto, un reto de las instituciones de educación superior en esta materia será buscar la integración de múltiples aspectos en la formación de formadores -axiológicos, disciplinares, de negocio, de innovación- y lograr, desde la transversalidad, el diálogo entre diferentes saberes, transformando así cierta ortodoxia imperante en la academia contemporánea. Una tarea esencial es fomentar en el estudiante un sentido integral de la vida, y ello parte el reto del conocimiento interdisciplinar, elemento vital en su proceso formativo, para culminar en un ejercicio transdisciplinar. Lo anterior, en suma, significa aprender a manejar una complejidad de saberes.

El ejercicio transdisciplinar implica una resignificación del conocimiento desde la gestión integral. En otras palabras, es fundamental una universidad al servicio de las necesidades y los problemas sociales reales presentes en la humanidad. La Organización de Naciones Unidas (ONU, 2015), en su Agenda 2030 para el nuevo desarrollo sostenible, explicita la importancia del emprendimiento para hacer posible el objetivo de desarrollo sostenible 4, a saber, garantizar una educación inclusiva, equitativa 
y de calidad, y promover oportunidades de aprendizaje durante toda la vida de todos. Así, una de las metas para este objetivo establece: “De aquí a 2030, aumentar considerablemente el número de jóvenes y adultos que tienen las competencias necesarias, en particular técnicas y profesionales, para acceder al empleo, el trabajo decente y el emprendimiento".

Aunque la formación en emprendimiento impartida por las instituciones de educación superior es diversa, en el presente se evidencia cierto acuerdo en la importancia de fomentar el espíritu y la cultura del emprendimiento en todos los campos disciplinares de formación. Lo anterior implica la consolidación de una gestión de la formación en estas instituciones para entregarle a la sociedad profesionales colaborativos y comprometidos con el desarrollo social. Por ello, una pregunta central emerge: ¿cuál es la gestión educativa que las instituciones de educación superior deberán adoptar para formar profesionales y emprendedores que contribuyan a resolver las necesidades humanas? Vega (2016), a este respecto, enuncia varias dificultades emergentes:

A grandes rasgos, se evidencia que existe un abordaje de la enseñanza del emprendimiento de forma fragmentada y que su desarrollo ha sido de forma lenta (Gibb, 2005). Por ello, desde la universidad es pertinente diseñar e implementar un modelo de formación del emprendimiento que motive al estudiante a tomar e implementar acciones emprendedoras. (p 37).

La visión del emprendimiento en las instituciones de educación superior se concibe como un sistema que tiene unas entradas: orientación directiva, estudiantes y plan curricular. Es un proceso que hace referencia a la pedagogía y didáctica de la educación del emprendimiento. Tiene también unas salidas: los resultados obtenidos del proceso. Tal concepción sistémica puede ampararse en el paradigma de la complejidad, puesto que el objeto de la enseñanza del emprendimiento es el ser humano, que ciertamente es complejo. Del mismo modo que impartir educación en emprendimiento de forma integral es un problema complejo, así también los resultados generados de la educación en emprendimiento conforman un hecho complejo que merece ser estudiado.

La teoría de la complejidad del sociólogo y antropólogo Edgar Morin brinda criterios y perspectivas para ser utilizados y explicar los componentes del emprendimiento, no solo desde su practicidad, enfoque, carácter y línea del empresarismo, sino desde su inmersión en el profundo conocimiento de las actitudes y aptitudes de lo humano. Morin (1996) Ilama la atención sobre una cuestión profunda: “¿Cuál 
es la sabiduría que hemos perdido en el conocimiento y cuál es el conocimiento que hemos perdido en la información?". En otras palabras, cuál es el conocimiento que no alcanzamos a percibir o que se pierde por el ritmo acelerado con el que se desarrollan los eventos. Según el autor, no es suficiente con conocer, sino que hay que integrar. No es suficiente con poseer la información, es necesario que el ser emprendedor genere una empatía social con su entorno, privilegiando las características humanas orientadas al servicio del bien común, sobre los conocimientos técnicos y el afán de lucro. Morin (1996) aborda muy bien la necesidad de esta integración, aunque tomando por caso la organización de una empresa:

[La] comprensión de la complejidad requiere un cambio muy profundo en nuestras estructuras mentales. El riesgo, si ese cambio de estructuras mentales no se produce, sería el de ir hacia la pura confusión o el rechazo de los problemas. No está el individuo por una parte, la sociedad por otra, la especie de un lado, los individuos del otro, de un lado la empresa con su organigrama, su programa de producción, sus estudios de mercado, del otro lado sus problemas de relaciones humanas, de personal, de relaciones públicas. Los dos procesos son inseparables e interdependientes. (pp. 78-79).
Asimismo, Morin (1996) hace énfasis en la necesidad de "enseñar a vivir, no a sobrevivir", porque, según él, los seres humanos son hijos e hijas del cosmos. Tal postura, en la perspectiva del emprendimiento, remite a pensar dos criterios: el primero hace referencia a la orientación de los emprendimientos hacia la creación de valor social, y no como una simple alternativa de subsistencia -emprendimiento de oportunidad vs. emprendimiento de subsistencia-. El segundo concierne a la necesidad de que cualquier tipo de emprendimiento sea respetuoso con el medioambiente.

Morin (1996) muestra la relacionalidad "de las partes al todo y del todo con las partes". En este sentido, la formación en emprendimiento es un todo compuesto por varias partes. Esta complejidad invita a formar al emprendedor desde la confluencia de múltiples saberes, para que así, en pleno conocimiento de esta diversidad, pueda encontrar su vocación y logre darles sistematicidad e interconexión a los proyectos específicos de emprendimiento. Ello desde luego implica que las instituciones de educación organicen y estructuren un proceso complejo para impartir el emprendimiento. Esto es, como se ha indicado, un modelo de formación en emprendimiento transdisciplinar.

Así como lo hizo el autor en mención, hay una pregunta por el significado de lo humano 
que deben hacerse quienes participan en la formación en emprendimiento, pues esta no debe enfocarse exclusivamente en formar "empresarios" que crean que su papel irrestricto en el mundo es lograr el lucro por el lucro, sin importar a quién se atropella y sin tener en cuenta la afectación en lo social y lo ambiental. En el otro extremo está la tendencia a confundir el emprendimiento con actividades organizadas y ejecutadas desde proyección social o como una alternativa de beneficencia social, pero donde el interés por generar progreso queda minimizado. Morin (1996) insistirá en la necesidad de abandonar la "idea simplista del progreso" propia de la tríada ciencia-técnica-industria, porque a esta se le atribuye el calificativo de "motor que impulsa los adelantos", lo cual no es cierto si allí hay un despojo de valores humanos -baste pensar cómo el desarrollo industrial ha provocado muchas veces guerra, hambre y destrucción-.

Podría señalarse, entonces, que la formación en emprendimiento estaría ubicada en medio de dos tendencias: una que promueve el emprendimiento orientando hacia el conocimiento y la generación de propuestas tecnológicas que desplazan la mano de obra, disminuyendo los costos de producción. La otra se orienta al desarrollo de emprendimientos de tipo social, aunque muchas veces ello se convierta en una actividad inmediata de beneficencia. Aunque la creatividad y la innovación son indudablemente necesarios y oportunos para la nueva sociedad del conocimiento, ningún tipo de formación en emprendimiento -incluidas las start-ups- debería dejar por fuera la inclusión de los valores del ser humano y, más aún un perfilamiento del emprendedor desde una estructuración compleja.

\section{INTERÉS Y ACCIÓN COMUNICATIVA: EL EMPRENDIMIENTO EN PERSPECTIVA HABERMASIANA}

Actuar con espíritu de emprendimiento pertenece a la esencia del ser humano; de algún modo se ha olvidado que esa intencionalidad de superar obstáculos y suplir necesidades no es un tema aislado de la historia, como tampoco lo concerniente a la conceptualización de las características y aptitudes emprendedoras desde las diferentes disciplinas. Además de la perspectiva de la complejidad para nutrir la comprensión del emprendimiento en las instituciones de educación superior, es posible dialogar con Habermas $(1982,1987,1981)$ para poner de relieve otros puntos de vista renovadores. El autor en mención hace un esfuerzo plausible por hallar las causas que han desplazado la teoría del conocimiento por una teoría de la ciencia, desde donde se ha generado un olvido de los estadios de la reflexión. 
Es cierto que el ser humano es histórico $y$, por tanto, produce un conocimiento que va evolucionando en el curso del tiempo. El sujeto se autorrealiza en la historia, se comunica, consensua, trabaja y, al tiempo, siempre ha deseado dominar lo natural. Es así como surge el objetivo de la teoría de los intereses cognoscitivos, que radicaliza lo epistemológico en el análisis del sujeto por y a partir de su autorrealización en el transcurso de la historia.

Habermas (1982) propone tres tipos de interés, y para cada uno de ellos plantea modos o estadios de investigación. El primer de ellos es el interés técnico, desde el estadio de las ciencias empírico-analíticas y respecto de lo cual McCarthy (1987) expresa:

La tesis de Habermas es que la orientación general, que guía las ciencias de la naturaleza, está basada en un interés de raíces antropológicas profundas, por la predicción y el control de sucesos que acontecen en el entorno natural. (p. 80).

Ahora bien, si se parte de la identificación del emprendimiento como realidad intrínseca del humano desde su creación y en cuanto ser histórico, entonces el emprendimiento estaría inmerso desde las experiencias por él vividas, cuyos procesos y evolución se cointegran con lenguajes y acciones. En ilación con este presupuesto, vale reiterar: el emprendimiento no puede constituirse en una práctica de corte netamente administrativo, contable, de mercadeo o financiero, incorporada en un proceso de evolución utilitarista y mercantilista. Si el emprendimiento se plantea desde la esencia del ser humano como la forma de pensar y actuar por medio del reconocimiento de las necesidades del entorno para la toma de decisiones acertadas, entonces desde el interés técnico de Habermas es factible verlo -así como las herramientas empresariales- como medio para mejorar en un momento dado las condiciones de los seres humanos en la tierra. En otras palabras, el emprendimiento sería reconocido como esencial al ser humano $y$, en conexión, se identificaría su presencia en las distintas disciplinas.

Así, desde el interés técnico, el campo del emprendimiento invitaría a la formulación de las siguientes preguntas: ¿cómo se podrían mejorar las condiciones de los seres humanos en el planeta?, ¿cómo despertar el interés por parte de los estudiantes en el reconocimiento de los diferentes entornos locales, regionales, nacionales e internacionales?, ¿cómo mejorar las condiciones de las personas que están en situación de vulnerabilidad? Adicionalmente, si se reconoce que el emprendimiento no es ajeno y está implícito en todas las áreas disciplinares existentes, las preguntas serían: ¿cómo des- 
de la gestión educativa se pueden orientar o aplicar los diferentes conocimientos técnicos?, ¿qué es lo que debe hacerse para aportar y beneficiar a la comunidad?, ¿en qué está bien o mal aplicar los conocimientos técnicos? Todo lo anterior tiene un componente intersubjetivo.

En segundo lugar se encuentra la orientación general, que se basa en el interés por la necesidad inexorable de entendimiento, de autoentendimiento y de comunicación, a lo que el autor llama interés práctico, contemplado en el estadio de las ciencias histórico-hermenéuticas. McCarthy (1987) afirma que en Habermas "la racionalidad del discurso sobre la adecuación de convenciones o de significados de conceptos no es la racionalidad de las operaciones efectuadas sobre procesos objetivados; implica interpretación de conceptos, de fines, de valores y de razones" (p. 92). La racionalidad de la que habla Habermas (1982) está mediada por aspectos socioculturales en los que intervienen la acción y necesidad de interpretación; pero para que se realice la interpretación, esta debe tener lugar con el otro o en comunidad. Por ello, la racionalidad no puede ser reducida a una simple acción instrumental. Es decir, el interés práctico posibilita el conocimiento objetivo, lo cual da como resultado un entendimiento intersubjetivo que evita que el ser humano sea considerado como una cosa $u$ objeto manipulable.
El interés práctico se podría considerar a partir de las necesidades y las relaciones humanas, en el sentido de cómo tendrían que convivir las personas en las actuales condiciones socioculturales y ambientales. Asimismo, se podría decir que el interés práctico es inherente a las actuaciones de los seres emprendedores, lo cual es corroborable al estudiar el pasado y el presente de las diferentes sociedades y sus líderes, y en la ruta en que se ha sabido trazar el futuro de las relaciones y la vida en comunidad.

El último es el interés emancipatorio, que se basa en las ciencias de orientación crítica, cuyo objetivo es descubrir los impulsos que intervienen sobre el ser humano y que impiden su libertad. En otras palabras, este interés ayuda al ser humano a tomar conciencia y causar su liberación. Según Habermas (1982), las teorías son sistemas de enunciados que pueden ser correctos o incorrectos, y en determinado momento pueden ser verificados o falseados. Por lo anterior, conviene formular: ¿qué revelan estos enunciados, cuando son validados, acerca de la realidad?

Analizado a partir del interés emancipatorio, el emprendimiento deberá permitir orientar al ser humano desde sus diferentes intereses técnicos y prácticos, haciendo que la formación técnica e histórica aplique en su conjunto la formación integral de un ser humano emprendedor, en función de hacer posible la 
producción del conocimiento y en libertad, para saber tomar decisiones incluyentes.

En segundo lugar, es posible encontrar en la Teoría de la acción comunicativa propuesta por Habermas (1981) otros horizontes de comprensión en el problema que acá nos compete. Desde esta propuesta, se pretende ver más allá de la técnica, porque busca observar, medir y analizar las acciones que se producen dentro de la comunidad. Habermas (1981) dirá respecto a la acción comunicativa:

Las acciones instrumentales pueden ir asociadas a interacciones sociales. Las acciones estratégicas representan, ellas mismas, acciones sociales. Hablo, en cambio, de acciones comunicativas cuando los planes de acción de los actores implicados no se coordinan a través de un cálculo egocéntrico de resultados, sino mediante actos de entendimiento. (p. 331).

La ética del discurso de este autor se orienta hacia la posibilidad de identificar los acuerdos racionales asociados a cuestiones de rectitud normativa y decisiones morales encaminadas a lograr acuerdos, buscando solución a problemas práctico-morales y su incidencia en la convivencia social. Esto, claro está, implica la realización de actos comunicativos en los que el entendimiento y los acuerdos son posibles si están basados en razones sobre cuestiones normativas. Este ejercicio teórico está centrado en un principio: "Solo pueden reivindicar lícitamente validez aquellas normas que pudiesen recibir la aquiescencia de todos los afectados en tanto que participantes en un discurso práctico" (MacCarthy, 1987, p. 16).

En el anterior axioma está expresado el principio de universalidad y se configura como un ejercicio intersubjetivo en el que todos los copartícipes expresan sus posiciones mediante argumentos. Es así como las personas que se comunican en el entorno social y cultural, según Habermas (1981), se comportan de cierta manera; en otros términos, se origina una acción comunitaria, un acto del entendimiento. Adicionalmente, el autor indica que la acción estratégica pretende utilizar a los seres humanos como medios para que determinada persona obtenga sus fines. Esto es, hay un cálculo de los beneficios e intereses desde lo personal o el egocentrismo. En cambio, la acción comunicativa se presenta cuando las personas dialogan y se dan a sí mismas unas normas mínimas de convivencia, siempre haciendo uso de la razón, para poder construir, crear y convivir en sociedad. Se originan entonces ciertos principios éticos de convivencia:

En las normas válidas, los resultados y los efectos secundarios que se deriven de su seguimiento universal para la satisfacción de 
los intereses de todos y cada uno tienen que poder ser aceptados por todos sin coacción alguna. (Habermas, 1987, p. 16).

Teniendo en cuenta lo anterior, la formación para el emprendimiento puede ser visualizada desde las instituciones de educación superior como sinónimo de una acción comunicativa, presente y pertinente a todas las áreas disciplinares. Un emprendedor, desde el marco hasta ahora expuesto, forma parte de una comunidad, en cuyo contexto el emprendimiento se consolidad de forma dialógica. Entendido así en la gestión de la formación en las instituciones de educación superior, es posible generar espacios de diálogo que permeen todas las disciplinas, permitiendo fijar acuerdos y estados de reflexión desde la misma gestión educativa, conducentes a generar acciones benéficas en los contextos sociales.

Los estudiantes, mediante la creatividad y la innovación, formularán propuestas emprendedoras desde el interés y beneficio general a partir de la acción técnica, práctica y emancipatoria, pero con características hacia el bienestar de la humanidad, con reconocimiento de lo histórico y la realidad de los entornos, con principios éticos, respeto por la libertad y el trabajo en comunidad. Interpretando a Habermas (1987), se podría decir que el emprendimiento ha estado presente en el actuar histórico del hombre, con quien se ha desarrollado; pero, al mismo tiempo, se ha desvirtuado su concepción, al hacerse posible y priorizarse desde acciones estratégicas de cálculo, intereses y beneficios instrumentales, destruyendo su significado de integralidad en los procesos de comunicación de las mismas acciones comunitarias.

Por ello, hoy es necesario analizar el emprendimiento desde la esencia misma del ser del sujeto, de su significado y componente de integralidad. No se puede ignorar la acción instrumental y estratégica que ha permeado el emprendimiento, pero se hace necesario generar una transformación en el pensamiento del hombre con respecto a este, partiendo desde los mismos líderes que ejercen la gestión educativa y organizacional en todos sus procesos. Lo anterior solo se puede dar con la acción comunicativa en razón, diálogo y mediación en cada una las disciplinas existentes.

\section{CONCLUSIONES}

Es común definir como emprendedores a quienes crean empresa, pero tal visión se ha ido dinamizando, entre controversias y aceptación, hasta derivar en teorías y proyectos específicos, como es el caso de la aplicación de leyes concretas para su reglamentación en varios países. Sin duda, hoy el emprendimiento ha adquirido mayor legitimidad académica y organizacional, pues potencializa la integración de distintos 
procesos. Además, desde la perspectiva de la creación de empresas, ha tenido nuevos espacios y teorías que le han concedido relevancia y pertinencia como objeto de estudio. Un abordaje desde la teoría de la complejidad de Edgar Morin y los intereses y la acción comunicativa de Habermas dan apertura a otras aristas de análisis y comprensión desde un horizonte más integral e interdisciplinar.

En las instituciones de educación superior, la formación del emprendimiento puede ser flexible en su composición curricular, porque contempla, además de las herramientas administrativas, múltiples saberes disciplinares: sociales, culturales y humanísticos. Allí se ponen en juego múltiples competencias que entrelazan y ponen en diálogo/tensión valores culturales, intereses y demandas al desarrollo de los estudiantes. Cualquier acción desde la gestión educativa desarrollada en las instituciones de educación superior, si contiene y aplica una formación para el emprendimiento con carácter transdisciplinar $-\mathrm{y}$ no solo desde el empresarismo-, le permitirá al estudiante ser partícipe de una formación en coherencia con sus intereses y necesidades, y también desde una dimensión más integral y dialógica de los saberes.

Es inminente la necesidad de seguir avanzando en el estudio de las causas reales que originan y motivan las características del ser emprendedor desde una formación y visión integral en las personas. Hay un campo abierto de estudio para aclarar el origen y desarrollo de las características que hacen que una persona se vuelva emprendedora.

Los diferentes actores involucrados en el campo del emprendimiento -especialmente en la gestión de la formación- deben buscar nuevas estrategias para repensar su acción de una manera colaborativa y desde las diversas disciplinas. El emprendimiento históricamente se ha desarrollado desde la mirada y perspectiva de las disciplinas administrativas y financieras, con sus distintos conocimientos y aportes, de manera cerrada. Hoy se reconoce la importancia de vincular y aprovechar los aportes de los diferentes campos disciplinares, mediante la creación de nuevas estrategias y formas de abordaje.

Se podría concebir el emprendimiento desde el factor humano y su visión desde el pensamiento complejo, en contraposición a quienes dicen que no es pertinente el emprendimiento al conocimiento científico.

Las instituciones de educación superior tienen el compromiso con la sociedad de generar nuevas visiones para articular el ecosistema del emprendimiento colombiano, identificando los esfuerzos que, de forma aislada, tanto en 
los ámbitos regional como local, vienen realizándose, en clave de gestar un colectivo que trabaje de manera coordinada en torno al emprendimiento.

Desde el pensamiento crítico de la teoría de Habermas, el consenso debe ser uno de los principales elementos presentes en el diálogo, que permitan el desarrollo y sostenimiento de centros de emprendimiento en las instituciones de educación superior. Este es también un principio que permite crear los espacios $\mathrm{y}$ ambientes emprendedores en estas instituciones donde no están constituidos, como también avanzar en la construcción de un marco teórico que permita comprender la interdependencia disciplinar y multidimensional del campo del emprendimiento.

\section{REFERENCIAS}

Congreso de la República de Colombia (2006). Ley 1014 de 2006, Ley de Fomento a la Cultura del Emprendimiento. Diario Oficial, 46,164 .

Cuervo, A. Ribeiro, D. y Roig, S (2006). Entrepreneurship, conceptos, teoría y perspectiva. Madrid, España: Fundación Bancaja.

Drucker, P. (1985). Innovation \& entrepreneurship: Practice and principles. Boston, USA: Butterworth Heinemann.

Drucker, P. (1999) Management challenges for the 21st century. Nueva York, USA: HarperCollins Publishers.
Duarte, T. y Ruiz, T. (2009). Emprendimiento, una opción para el desarrollo. Scientia et Technica, 15(43), 326331.

Ekelund, R. y Hébert, R. (1992). Historia de la teoría económica y de su método. Madrid, España: McGraw-Hill.

Girón, A. (2010). Schumpeter: aportaciones al pensamiento económico. Recuperado en: http://revistas. bancomext.gob.mx/rce/magazines/41/7/RCE.pdf

Habermas, J (1981). Teoría de la acción comunicativa. Fráncfort, Alemania: Trotta.

Habermas, J (1982). Conocimiento e interés. Madrid, España: Taurus.

Habermas, J. (1987). Teoría de la acción comunicativa. Madrid, España: Taurus.

Malthus, T. (1970). Ensayo sobre el principio de la población. Ciudad de México, México: Fondo de Cultura Económica.

Matiz, F. (2006). Investigación en emprendimiento, un reto para la construcción de conocimiento. Revista EAN, 66, 169-182.

McCarthy, T. (1987). La teoría crítica de Jürgen Habermas. Madrid, España: Tecnos. 
Ministerio de Educación Nacional (MEN) y el Instituto Colombiano para el Fomento de la Educación Superior (ICFES). (2001). Estándares mínimos de calidad para la creación y funcionamiento de programas universitarios de pregrado. Bogotá, Colombia: MEN

Morin, E. (1996). Introducción al pensamiento complejo. Barcelona, España: Gedisa.

Morin, E. (2000). Los siete saberes necesarios para la educación del futuro. París, Francia: Unesco.

Morin, E. (2010). Sobre la interdisciplinariedad. Recuperado en: https://www.icesi.edu.co/revistas/ index.php/publicaciones_icesi/article/view/643

Organización de Naciones Unidas (ONU) (2015). Objetivos de Desarrollo Sostenible. Recuperado en: https:// nacionesunidas.org.co/ods/
Osorio, F., Gálvez, É. y Murillo, G. (2010). La estrategia y el emprendedor: diversas perspectivas para el análisis. Cuadernos de Administración, 43, 65-80.

Rodríguez, A. (2009). Nuevas perspectivas para entender el emprendimiento empresarial. Pensamiento \& Gestión, 26.

Schumpeter, J. A. (1963). Teoría del desarrollo económico. Ciudad de México, México: Fondo de Cultura Económica.

Smith, A. (1987). Investigación sobre la naturaleza y causa de la riqueza de las naciones. Barcelona, España: Oikos Tau.

Taylor, F. (1961). Principios de la administración científica. Ciudad de México, México: Herrero Hermanos.

Vega Guerrero, J. (2016). Modelo de formación en emprendimiento social, para instituciones de educación superior en Colombia. Revista Escuela de Administración de Negocios, 81, 29-44. 